\title{
Optimal Power Flow Control Based Shunt FACTS Devices Using PSO Algorithm
}

\author{
التحكم الأمثل في سريان القدرة بواسطة أجزةة نظم النقل المرنة بإستخدام طريقة سرب الطيور الأمثل \\ Ekramy Saad*, R. A. Amer $^{* *}$ and G. A. Morsy ${ }^{* *}$ \\ "Egyptian Electricity Transmission Co., Alex. Zone, Egypt \\ *** Department of Electrical Engineering, Faculty of Engineering, Minoufiya University, Shebin El-
}

Kom, Egypt

\begin{abstract}
:
In this paper, an efficient and reliable evolutionary-based approach is employed to solve the optimal power flow (OPF) problem. This approach utilizes the global and local exploration capabilities of particle swarm optimization (PSO) to search for optimal setting of control variables for the OPF which is formulated as a nonlinear constrained objective optimization problem with both equality and inequality constraints. To illustrate how the proposed method has an efficient role, the standard IEEE 30-bus power system is studied and the PSO is used to minimize each of the total fuel cost, system power loss and voltage deviations. Two different types of FACTS devices are embedded with the test system, SVC and STATCOM, to achieve the objectives functions under certain constraints. The results show the effectiveness of STATCOM with optimal settings over the SVC with same conditions.

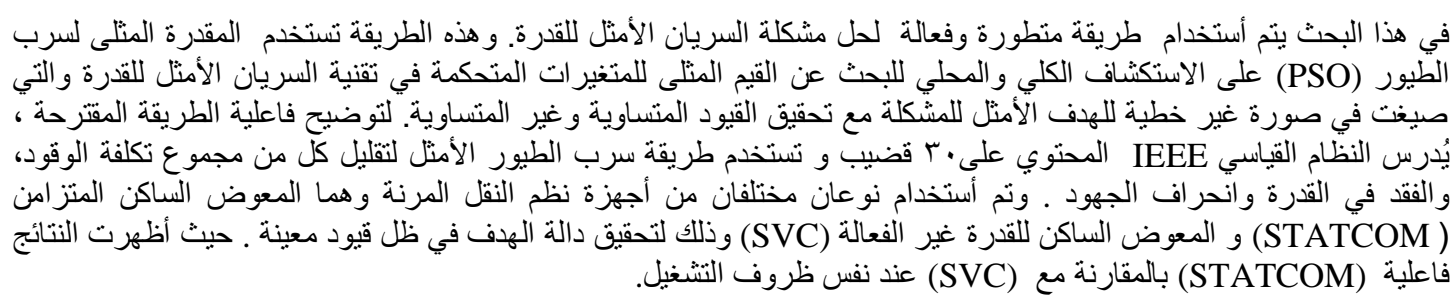

Key words - optimal power flow, particle swarm optimization, FACTS.

\section{INTRODUCTION}

The electrical energy demand increases continuously from time to time. This increase should be monitored or observed because few problems could appear with the power flows through the existing electric transmission networks. If this situation fails to be controlled, some lines located on the particular paths might become overloaded [1]. Due to the overloaded conditions the transmission lines will have to be driven close to or even beyond their transfer capacities. Building a new transmission line will not be an efficient way to solve these problems since it is quite complicated and due to the environmental and political reasons. One possible solution to improve the system operation was the use of Flexible AC Transmission Systems (FACTS) technologies. It opens up new opportunities for controlling the power, decreasing the losses and enhancing the unstable capacity of existing transmission lines [1].

FACTS devices can control power flow parameters such as series impedance, voltage, phase angle and so can reduce flow of heavily loaded lines and support voltage [2]. Also, FACTS devices can enhance transmission system control and increase line loading in some cases all the way up to thermal limits thereby without compromising reliability. These devices can be an alternative to reduce the flows in heavily loaded lines, resulting in an increased loadability, low system loss, improved stability of the network, reduced cost of production and fulfilled contracture requirement by controlling the power flows in the network [3].

FACTS include static compensators implemented with thyristors and self-commutated converters. Apart from thyristors-based controllers include devices such as static VAR compensator (SVC), thyristor controlled series capacitor (TCSC)...etc. On the other hand, the voltage source converter (VSC)-based devices includes static compensator (STATCOM), the unified power flow controller (UPFC) and static synchronous series compensator (SSSC). The voltage source converter (VSC) based controllers have superior performance over the thyristor-based controllers [4].

FACTS technology has introduced new, yet effective, ways of controlling a power system, which makes the system operation more flexible and secure. This is accomplished by targeting/modifying the key elements in power flow, namely transmission line impedance, phase angle and voltage magnitude [5].

Optimal Power Flow (OPF) problem in electrical power systems is considered as a static, non-linear, multiobjective or a single objective optimization problem. As 
the power industrial companies have been moving into a more competitive environment, OPF has been used as a tool to define the level of the inter utility power exchange [6]. The main objective of an OPF strategy is to determine the optimal operating state of a power system by optimizing a particular objective while satisfying certain specified physical and operating constraints [7].

A number of conventional optimization techniques have been applied to solve the OPF problem. They include linear Programming (LP), nonlinear programming (NLP), quadratic programming (QP), and interior point methods. All these techniques rely on convexity to find the global optimum [7].

During the history of science of computational intelligence, many evolutionary algorithms were proposed having more or less success in solving various nonlinear engineering optimization problems. Among them, the best are considered to be the popular particle swarm optimization (PSO) introduced by Kennedy and Eberhart [8].

The authors of [1] have presented the application of evolutionary computation technique for loss minimization and UPFC installation cost. In Ref. [5], FACTS devices were optimally allocated in power system to achieve the optimal power flow solution. The location of FACTS devices and the setting of their control parameters were optimized by bacterial swarming algorithm to improve the network performance. The authors of [6] were implementing a single OPF objective function optimization algorithm based on the particle swarm optimization (PSO), where the optimum generation pattern has been calculated as well as all control variables in order to minimize reactive loss together with meeting the transmission system limitations.

In paper [7], a dynamic and flexible hybrid approach in solving the OPF problem in the presence of multiple dynamic shunt compensators by fuzzy controller genetic algorithm has been proposed. Authors of [8], present a proposed PSO algorithms as well as the state-of-the-art PSO and the conventional interior-point OPF-based algorithm competed in the optimization problems of reactive power and voltage control. The results obtained indicated an improved performance of the proposed PSO algorithms.. Simulation is carried out on the IEEE 30-bus realtest-system (RTS). Simulation results demonstrated that the proposed evolutionary program (EP) technique is feasible for loss minimization scheme in other power system network. In [9], the optimal placement of FACTS controllers and their potential role in reactive power market has been studied. Considerable improvement in reactive power marginal prices (RPMP) has been obtained with SVC. As the number of SVCs is increased to four, the impact on reactive marginal prices and objective functions are improved also. The proposed model is applied on the IEEE 39-bus system.

In Ref. [10], the author uses particle swarm optimization (PSO) algorithm for optimal settings of optimal power flow problem control variables. The proposed approach has been examined and tested on the standard IEEE 30bus test system. Different objective functions have been considered to minimize the fuel cost, to improve the voltage profile and to enhance voltage stability.

This paper presents the use of PSO algorithm for solving the OPF problem which satisfies each of equality and inequality constrains. The objectives are minimizing each of the total fuel cost, the total power loss and voltage deviations. The proposed method will be applied on the standard IEEE 30-bus power system, where two different types of shunt FACTS devices are attached to the tested system. SVC and STATCOM are used to control the system variables using PSO algorithm to achieve the objectives functions. The simulation results are obtained in comparative form to show the effectiveness of the proposed method.

\section{FACTS DEVICES}

It is known that power transfer limit and quality of supply can be drastically improved by insertion of voltage or current into a power system. This can be achieved by use of power electronics switches or converters. The technology is called Flexible AC Transmission system (FACTS) [11]. A major thrust of FACTS technology is the development of power electronics based systems that provide dynamic control of the power transfer parameters transmission voltage, line impedance and phase angle without generation rescheduling or topological change. Different categories of FACTS controllers can be used in [9].

\section{2-1. Static Var Compensation (SVC)}

The installation of shunt compensators in a transmission system aims ultimately at increasing the transmittable power, which might be required for improving overall system stability as well as transmission characteristics. The Static Var Compensator (SVC) equipment is composed by capacitors, thyristors and inductances. $\mathrm{SVC}_{\mathrm{s}}$ have the ability to either absorb or inject reactive power when needed and such accomplish the task of voltage and power transfer regulation [5]. There are two ways for modeling these devices. The first model considers SVC as variable impedance, which is adapted automatically to achieve the voltage control. This is called the passive model and its main disadvantage is the changing of nodal admittance matrix whenever there is a variation in the operation conditions of the power grid. The second model, called active model, represents SVC as a nodal power injection. It uses active sources in the equivalent circuit, which can be easily incorporated in the OPF calculations [12]. In this work, the SVC modeled as an ideal reactive power injection. For the connection of steady state model of SVC in a particular 
load bus " $i$ ", the exchanged reactive power at that bus is $Q_{i}=Q_{s v c}$. The $S V C$ equivalent circuit is shown in Fig.1.

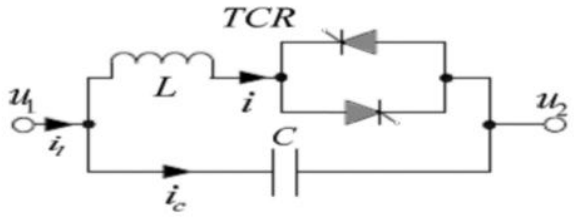

Fig. 1 Equivalent circuit of SVC

\section{2-2. Static Synchronous Compensator (STATCOM)}

STATCOM is a shunt compensation device which can be used for improving the voltage profile. It is a shunt controller and it injects current to the transmission line. When the system voltage is greater than generator voltage, it absorbs the reactive power, and if smaller then it generates the reactive power. It can be based on both voltage source and current source convertor. It can be designed to be an active filter to absorb system harmonics [13].

STATCOMs have better characteristics than SVCs. When the system voltage drops sufficiently to force the STATCOM output to its ceiling, its maximum reactive power output is not affected by the voltage magnitude. Therefore, the STATCOM exhibits constant current characteristics when the voltage is below the limit. Fig. 2 shows the STATCOM schematic diagram and equivalent circuit.

The bus at which the STATCOM is connected is represented as a PV bus, this dispositive can be generated or absorbed reactive power would reach to the maximum limit. The losses in the STATCOM are neglected and STATCOM is assumed be purely reactive [14].

The STATCOM provides operating characteristics similar to a rotating synchronous compensator without the mechanical inertia. The STATCOM provides rapid controllability of the three phase voltages, both in magnitude and phase angle. The STATCOM basically consists of a step-down transformer with a leakage reactance, a three-phase GTO or IGBT voltage source inverter (VSI), and a DC capacitor. The AC voltage difference across the leakage reactance produces reactive power exchange between the STATCOM and the power system, such that the AC voltage at the bus bar can be regulated to improve the voltage profile of the power system, which is the primary duty of the STATCOM. However, for instance, a secondary damping function can be added into the STATCOM for enhancing power system oscillation stability [15].

Active power flow is determined by the phase angle difference between the sources and the reactive power flow is determined by the voltage magnitude difference between the sources. Hence, STATCOM can control reactive power flow by changing the fundamental component of the converter voltage with respect to the $\mathrm{AC}$ bus bar voltage both phase wise and magnitude wise [16].

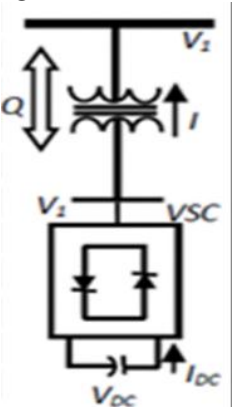

(a)

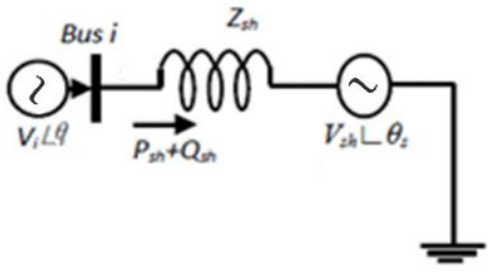

(b)
Fig. 2 a) Basic configuration of a STATCOM

b) STATCOM equivalent circuit

\section{OPF FORMULATION}

The main objective of the OPF is to obtain an optimal operating point, usually linked to economic variables, subject to a set of technical constraints imposed by the power grid. Mathematically, it can be written as a nonlinear optimization problem as follows:

$\begin{array}{lc} & \text { Minimize } f(x) \\ \text { Subject to: } & g(x)=0 \\ & h(x) \leq 0\end{array}$

where, $f(x)$ is the objective function, $\mathrm{g}(\mathrm{x})$ and $\mathrm{h}(\mathrm{x})$ are the set of equality and inequality constraints respectively. $\mathrm{x}$ is the vector of control and state variables. The control variables are generator active and reactive power outputs, bus voltages and transformers tap-setting. The state variables are voltage and angle of load buses.

\subsection{Objectives Functions}

Three different objectives will be considered in this paper, these are fuel cost, total active power losses and voltage deviation, as follows:

a) Fuel cost

For optimal active power dispatch, the objective function $f$ is total generation cost expressed as follows:

Min $f_{1}=\sum_{i=1}^{N_{g}} a_{i}+b_{i} P_{g}+c_{i} P_{g}^{2}$

where, $N_{g}$ is the number of thermal units, $P_{g i}$ is the active power generation at unit $\mathrm{i}$ and $a_{i}, b_{i}$ and $c_{i}$ are the cost coefficients of the $i^{\text {th }}$ generator, the values of these coefficients are given in Table 1.

Table 1 Generator cost coefficients.

\begin{tabular}{|c|c|c|c|c|c|c|}
\hline \multirow{2}{*}{ Coefficients } & \multicolumn{7}{|c|}{ Generator } \\
\cline { 2 - 7 } & $\mathrm{G}_{1}$ & $\mathrm{G}_{2}$ & $\mathrm{G}_{3}$ & $\mathrm{G}_{4}$ & $\mathrm{G}_{5}$ & $\mathrm{G}_{6}$ \\
\hline $\mathrm{a}$ & 0.0 & 0.0 & 0.0 & 0.0 & 0.0 & 0.0 \\
\hline $\mathrm{b}$ & 200 & 175 & 100 & 325 & 300 & 300 \\
\hline $\mathrm{c}$ & 37.5 & 175 & 625 & 83.4 & 250 & 250 \\
\hline
\end{tabular}

b) Power loss

The second objective is minimizing the total power loss in the transmission network, which is defined as follows: 
$\operatorname{Min}_{f_{2}}=\sum_{k=i}^{n l} g_{k}\left[V_{i}^{2}+V_{j}^{2}-2 V_{i} V_{j} \cos \theta_{i j}\right]$

(5)

where $g_{k}$ is the conductance of branch between bus $i$ and bus $j, V_{i}$ and $V_{j}$ are the voltages at buses $i$ and $j$.

\section{c) Voltage deviation}

The third objective is minimizing the voltage deviation at all busses, and it can express by the following equation:

$$
\text { Min } \quad f_{3}=\sum_{i=1}^{n p q}\left|V_{i}-V_{\text {ref }}\right|
$$

where, $n p q$ is the set number of load bus, $V_{\text {ref }}$ is the set voltage reference (1.0 p.u.).

\subsection{Constraints}

The equality constraints $g(x)$ are the power flow equations, expressed as follows:

$$
P_{g i}-P_{d i}=\sum_{j=1}^{N}|V i\|V j\| Y i j| \cos (\delta i-\delta j-\delta i j)
$$$$
Q_{g i}-Q_{d i}=\sum_{j=1}^{N}|V i\|V j\| Y i j| \operatorname{Sin}(\delta i-\delta j-\delta i j)
$$

The inequality constraints $h(x)$ reflect the limits on physical devices in the power system as well as the limits created to ensure system security, such the limits on active and reactive generations, the bounds on the tap ratio $(\mathrm{t})$, the upper limit on the active power flow $\left(P_{i j}\right)$ of line $\mathrm{i}-\mathrm{j}$, the bounds in bus voltage magnitudes, the bounds in Shunt FACTS parameters and these can be expressed as follows:

$$
\begin{aligned}
& P_{g i}^{\min } \leq P_{g i} \leq P_{g i}^{\max }, Q_{g i}^{\min } \leq Q_{g i} \leq Q_{g i}^{\max } \\
& \boldsymbol{t}_{i j}^{\min } \leq \boldsymbol{t}_{i j} \leq \boldsymbol{t}_{i j}^{\max } \\
& \left|\boldsymbol{P}_{i j}\right| \leq \boldsymbol{P}_{i j}^{\max } \\
& V_{i}^{\min } \leq V_{i} \leq V_{i}^{\max } \\
& Q^{\text {min }}<Q_{\text {FACTS }}<Q^{\max }
\end{aligned}
$$

\section{Particle Swarm Optimization (PSO)}

\subsection{PSO OVERVIEW}

Particle Swarm Optimization (PSO) is a relatively new evolutionary algorithm that may be used to find optimal solutions to numerical and qualitative problems. Particle Swarm Optimization was originally developed by James Kennedy and Russell Eberhart in 1995, and emerged from earlier experiments with algorithms that modeled the flocking behavior seen in many species of birds [1].

PSO is well known and popular search strategy that has gained widespread appeal amongst researchers and has been shown to offer good performance in a variety of application domains, with potential for hybridization and specialization. It is a simple and robust strategy based on the social and cooperative behavior shown by various species like flocks of bird, schools of fish. PSO and its variants have been effectively applied to a wide range of real life optimization problems [13].

In simulations, birds would begin by flying around with no particular destination and spontaneously formed flocks until one of the birds flew over the roosting area. Due to the simple rules the birds used to set their directions and velocities, a bird pulling away from the flock in order to land at the roost would result in nearby birds moving towards the roost. Once these birds discovered the roost, they would land there, pulling more birds towards it, and so on until the entire flock had landed. Finding a roost is analogous to finding a solution in a field of possible solutions in a solution space. The manner in which a bird who has found the roost, leads its neighbors to møve towards it, increases the chances that they will also find it.

This is known as the "socio-cognitive view of mind". The "socio-cognitive view of mind" means that a particle learns primarily from the success of its neighbors. The concept of the PSO consists of, at each time step, changing the \&elocity of (accelerating) each particle toward its $\mathrm{P}_{\text {best }}$ and $\mathrm{L}_{\text {best }}$ locations (local version of PSO). Acceleration is weighted by a random term, with separate random numbers being generated for acceleration toward $\mathrm{P}_{\text {best }}$ and $\mathrm{L}_{\text {best }}$ locations.

In the past several years, PSO has been successfully applied in many research and application areas. It is demonstrated that PSO gets better results in a faster, cheaper way compared [10].

\subsection{PSO algorithm}

The basic terms used in PSO technique are stated and defined as follows:

1. Particle $\mathbf{X}$ (I): It is a candidate solution represented by a $\mathrm{k}$-dimensional real-valued vector, where $\mathrm{k}$ is the number of optimized parameters. At iteration $\mathrm{i}$, the $\mathrm{j}^{\text {th }}$ particle $\mathrm{X}(\mathrm{i}, \mathrm{j})$ can be described as:

$\mathrm{X}_{\mathrm{i}}(\mathrm{i})=\left[\mathrm{X}_{\mathrm{j} 1}(\mathrm{i}) ; \mathrm{X}_{\mathrm{j} 2}(\mathrm{i}) ; \ldots . . \mathrm{X}_{\mathrm{jk}}(\mathrm{i}) ; \ldots . . \mathrm{X}(\mathrm{i})_{\mathrm{jd}}\right]$

Where: $X^{\prime}$ s are the optimized parameters and $d$ represents number of control variables

2. Population: It is basically a set of $n$ particles at iteration $\mathrm{i}$.

pop (i) $=\left[X_{1}(i), X_{2}(i), \ldots \ldots \ldots X_{n}(i)\right]^{T}$

Where: $\mathrm{n}$ represents the number of candidate solutions.

3. Swarm: Swarm may be defined as an apparently disorganized population of moving particles that tend to cluster together while each particle seems to be moving in a random direction. 
4. Particle velocity V(i): Particle velocity is the velocity of the moving particles represented by a ddimensional real valued vector. At iteration $i$, the $j^{\text {th }}$ particle $\mathrm{V}_{\mathrm{j}}(\mathrm{i})$ can be described as:

$\mathrm{V}_{\mathrm{j}}(\mathrm{i})=\left[\mathrm{V}_{\mathrm{j} 1}(\mathrm{i}) ; \mathrm{V}_{\mathrm{j} 2}(\mathrm{i}) ; \ldots . . \mathrm{V}_{\mathrm{jk}}(\mathrm{i}) ; \ldots . . \mathrm{V}_{\mathrm{jd}}(\mathrm{i})\right]$ where:

$\mathrm{V}_{\mathrm{jk}}(\mathrm{i})$ is the velocity component of the $\mathrm{j}^{\text {th }}$ particle with respect to the $\mathrm{k}^{\text {th }}$ dimension.

5. Inertia weight $\mathbf{w}(\mathbf{i})$ : It is a control parameter, which is used to control the impact of the previous velocity on the current velocity. Hence, it influences the trade-off between the global and local exploration abilities of the particles. For the initial stages of the search process, large inertia weight to enhance the global exploration is recommended while it should be reduced at the last stages for better local exploration. Therefore, the inertia factor decreases linearly from about 0.9 to 0.4 during a run. In general, this factor is set according to the following equation:

$\mathrm{W}=\mathrm{W}_{\text {max }}\left(\left(\mathrm{W}_{\text {max }}-\mathrm{W}_{\text {min }}\right) /\right.$ iter $\left._{\max }\right) \times$ iter

where, iter max $_{\max }$ is the maximum number of iterations and iter is the current number of iterations.

6. Individual best $X^{*}(\mathbf{i})$ : When particles are moving through the search space, it compares its fitness value at the current position to the best fitness value it has ever reached at any iteration up to the current iteration. The best position that is associated with the best fitness encountered so far is called the individual best $\mathrm{X}^{*}(\mathrm{i})$.

For each particle in the swarm, $X^{*}(\mathrm{i})$ can be determined and updated during the search.

For the $\mathrm{j}^{\text {th }}$ particle, individual best can be expressed

as:

$\mathrm{X}_{\mathrm{j}}(\mathrm{i})=\left[\mathrm{X}_{\mathrm{j}, 1} *(\mathrm{i}), \mathrm{X}_{\mathrm{j}, 2} *(\mathrm{i}), \ldots \ldots \ldots . . . \mathrm{X}_{\mathrm{j}, \mathrm{d}} *(\mathrm{i})\right]$

In a minimization problem with only one objective function $\mathrm{f}$, the individual best of the $\mathrm{j}^{\text {th }}$ particle $\mathrm{X}_{\mathrm{j}}^{*}(\mathrm{i})$ is updated whenever $f\left(\mathrm{X}_{\mathrm{j}}^{*}(\mathrm{i})\right)<f\left(\mathrm{X}_{\mathrm{j}}^{*}(\mathrm{i}-1)\right)$. Otherwise, the individual best solution of the $\mathrm{j}^{\text {th }}$ particle will be kept as in the previous iteration.

7. Global best $\mathbf{X}^{* *}(\mathbf{t})$ : Global best is the best position among all of the individual best positions achieved so far.

8. Stopping criteria: Termination of the search process will take place whenever one of the following criteria is satisfied:

a) the number of the iterations since the last change of the best solution is greater than a specified number.

b) the number of iterations reaches the maximum allowable number.

The particles are manipulated according to the following equations:

$V_{j d}=V_{j d}+c_{1} r_{1}\left(P_{j d}-X_{j d}\right)+c_{2} r_{2}\left(P_{g d}-X_{j d}\right)$

(14)
$X_{j d}=X_{j d}+V_{j d}$

(15)

where, $g$ is the best particle among all particles, $c_{1}$ and $c_{2}$ are positive constant and $r_{1}$ and $r_{2}$ are uniformly distributed numbers in $(0,1)$.

\subsection{Advantages of PSO}

Many advantages of PSO over other traditional optimization techniques can be summarized as follows [1]: PSO is a population-based search algorithm. This property ensures PSO to be less susceptible in being trapped on local minima. PSO makes use of the probabilistic transition rules and not deterministic rules. Hence, PSO is a kind of stochastic optimization algorithm that can search a complicated and uncertain area. This makes PSO more flexible and robust than conventional methods. PSO can easily deal with nondifferentiable objective functions because PSO uses payoff (performance index or objective function) information to guide the search in the problem space. Additionally, this property relieves PSO of assumptions and approximations, which are often required by traditional optimization models. PSO has the flexibility to control the balance between the global and local exploration of the search space. This unique feature of a PSO overcomes the premature convergence problem and enhances the search capability which makes it different from Genetic Algorithm (GA) and other heuristic algorithms.

\subsection{PSO implementation}

The proposed PSO based approach was implemented using Intel core i3 $1.0 \mathrm{GHz}$ processor with $4 \mathrm{~GB}$ of RAM in MATLAB 10.0. Initially, several runs have been done with different values of the PSO key parameters such as inertia weight and the maximum allowable velocity. Other parameters are selected as number of particles $\mathrm{n}=25$, the iterations $=100$, the inertia weight factor varied between $0.9-0.4$ and the cognitive and the social parameters $C_{1}$ and $C_{2}$ which are be equalized $\left(C_{1}=C_{2}=2\right)$, and $\operatorname{rand}_{1} \& \operatorname{rand}_{2}$ are random numbers uniformly distributed within (0-1).

\section{Simulation AND Numerical Results}

The proposed algorithm is implemented and tested on a standard IEEE 30-bus power system, it consists of sixgenerators located at buses $1,2,3,4,5$, and 6,41 branches (lines) and four transformers in line 9-13, 1013, 8-12, and 28-27 as shown in Fig. 3 [17]. The total active power load is $189.2 \mathrm{MW}$ while the total reactive power load is 126.2 MVar. The base power is taken as 100 MVA. For the studies, the PV and slack bus voltages between 0.95 to 1.15 , The algorithms have been implemented using the MATLAB programming language, detailed analyses of the results are presented and discussed in this section.

Three different cases studies are considered, in the first case study, only to verify the effectiveness of the PSO 
algorithm without considering the FACTS Controllers. In second case, the optimal power flow is treated considering a SVC which connected in shunt in the system and in the third case another device of shunt FACTS controllers "STATCOM" is considered. Minimizing each of "fuel cost", "total active power losses" and "voltage deviation", are the objectives which will be studied in each of three cases.

\subsection{Case 1: OPF using PSO without FACTS}

In this case the PSO algorithm is applied in the system under study to achieve the optimal power flow.

Figs. 4, 5 and 6 show the variations of total fuel cost, active power losses and summation of voltage deviation for the original power system without connecting any FACTS. Referring to these figures, it can be observed that the optimized fuel cost value is $801.1115 \mathrm{\$} / \mathrm{h}$, the optimized power loss is $3.2 \mathrm{MW}$ and the obtained voltage deviation equal 0.0311 . The optimal setting of control variable is recorded in Table 2.

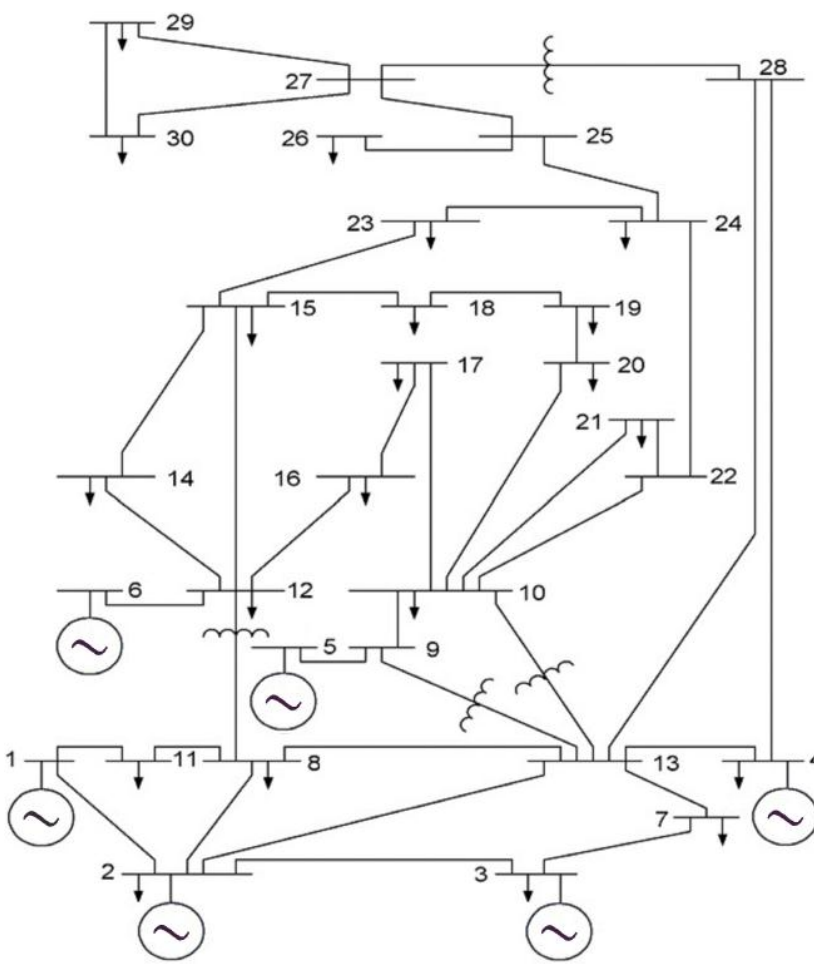

Fig. 3 The single line diagram of IEEE 30 bus test system

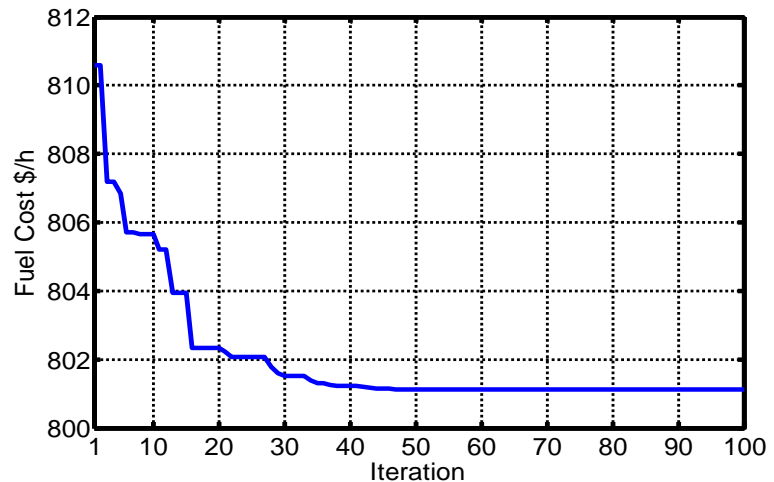

Fig. 4 The fuel cost variation of case 1

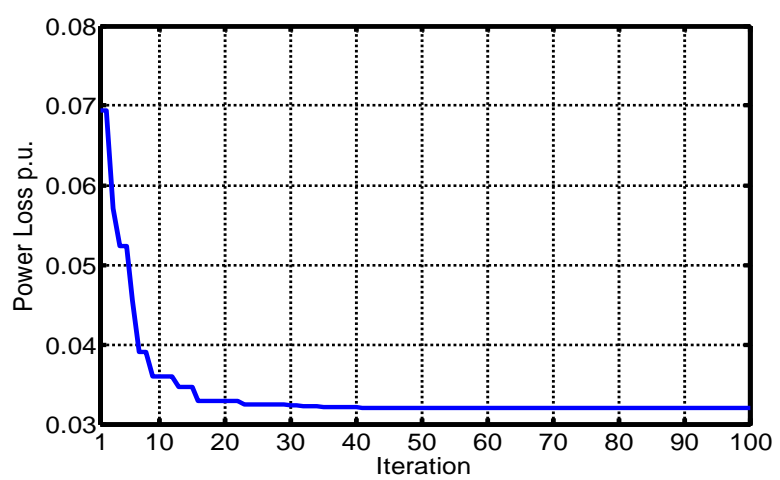

Fig. 5 The power loss variation of case 1

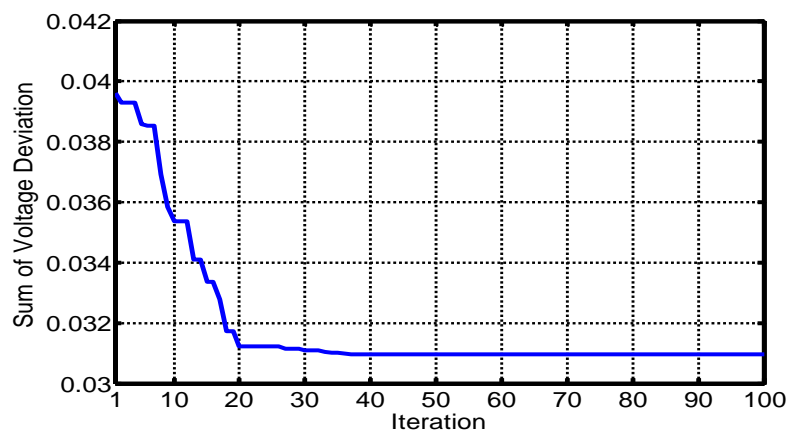

Fig. 6 The voltage deviation variation of case 1

\subsection{Case 2: OPF using PSO considering SVC}

In this case, a SVC is considered in OPF problem on the tested system. The optimal location of the SVC device is determined by the PSO algorithm to achieve the objectives functions. It is found that the optimal location for SVC at buses 8 which connected in shunt to minimize the fuel cost. The variation of the total fuel cost is shown in Fig. 7. Referring to this figure, the total cost is optimized to $800.627 \$ / \mathrm{h}$. The optimal setting of control variable is recorded in Table 2. 


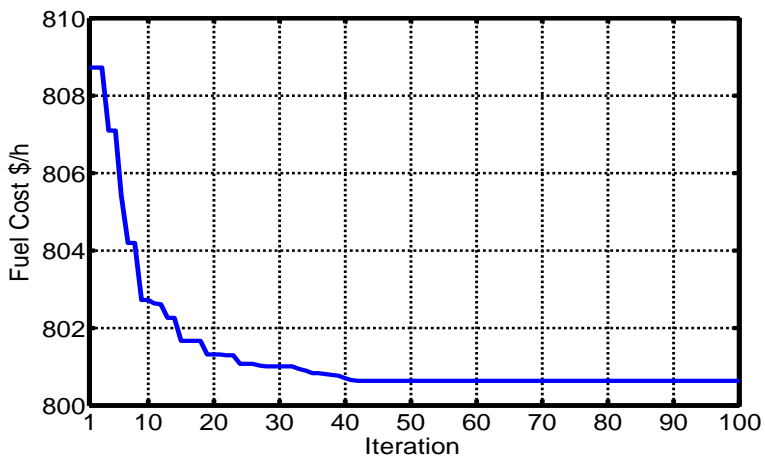

Fig. 7 The fuel cost variation of case 2

To minimize the total active power losses in transmission network, it is found that the best location of SVC device at bus 8 , which connected in shunt. The variation of the total power loss is shown in Fig. 8. Referring to this figure, the total power loss is optimized to $3.12 \mathrm{MW}$.

It is found that the best allocation of the SVC which connected in shunt at bus 8. Figure 9 shows the variation of voltage deviation. Referring to this figure, it can be observed that the voltage deviation is minimized

to

0.0275 .

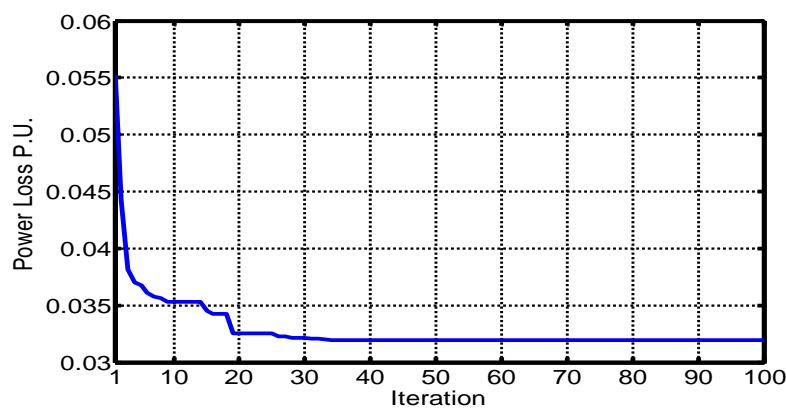

Fig. 8 The power loss variation of case 2

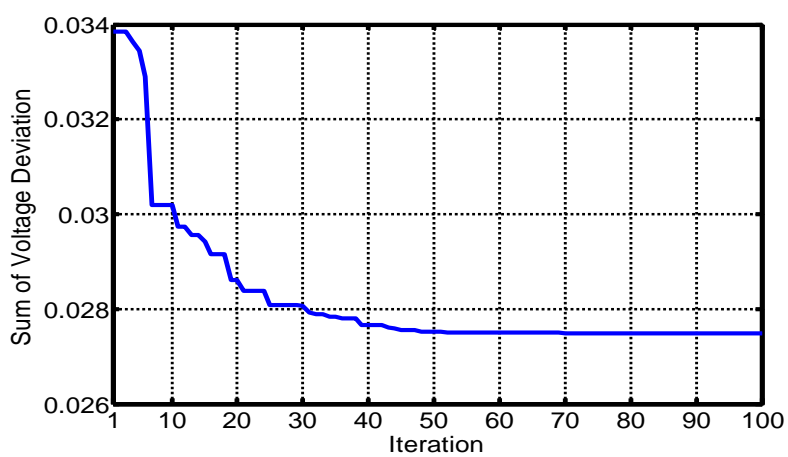

Fig. 9 The voltage deviation variation of case 2

The system voltage profile considering SVC device and it is compared to that of case las shown in Fig. 10. It is evident that the voltage profile is greatly improved compared to that of case 1 .

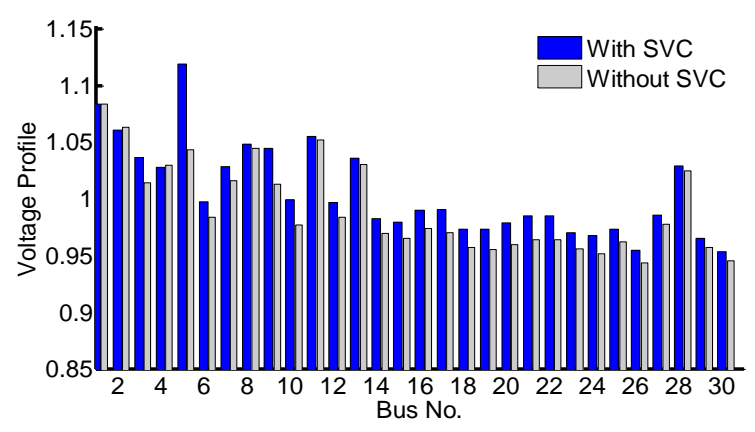

Fig. 10 The system voltage profile considering SVC

5.3 Case 3: OPF using PSO considering STATCOM In this case, another type of shunt FACTS devices embedded with the system under study. To determine the optimal allocation of STATCOM, PSO algorithm is used. It is found that the best location at bus 10. Fig. 11 shows the fuel cost variation of case 3 . Referring to this figure, it can be concluded that existence of STATCOM at suitable placement achieve the least value of fuel cost compared with the previous two studied cases.

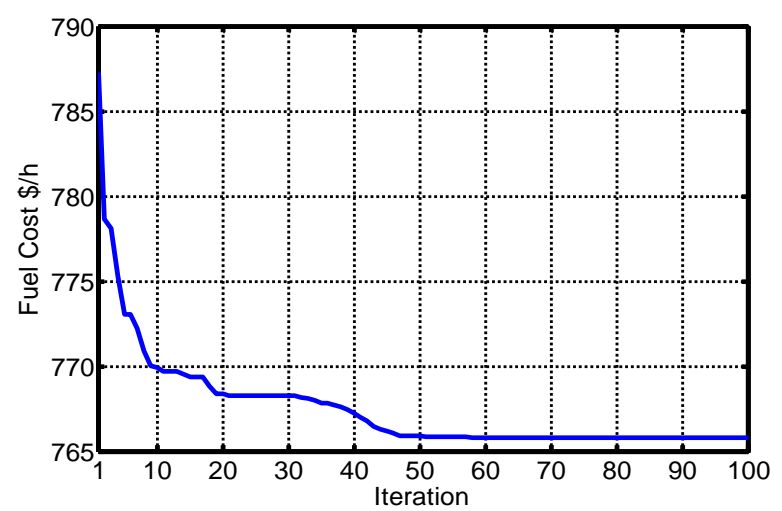

Fig. 11 The fuel cost variation of case 3

The effect of existence of STATCOM to minimize the total active power losses for the studied system is shown in Fig. 12. From this figure, it can be observed that the value of power loss is more minimized compared to that in case 2. The optimal setting of control variable is recorded in Table 2 .

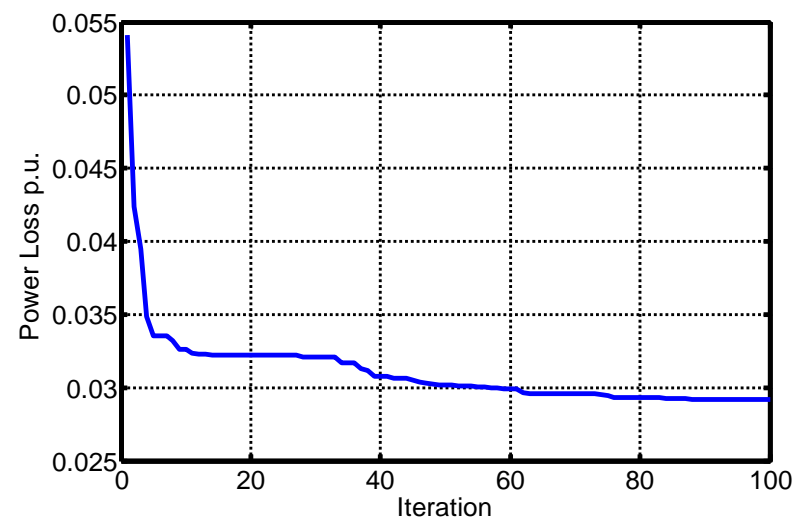

Fig. 12 The power loss variation of case 3 
Fig. 13 shows the effect of existence of STATCOM on the variation of voltage deviation at all buses in the system. It is evident that the summation of voltage deviation is more minimized compared to those of case 1 and case 2 .

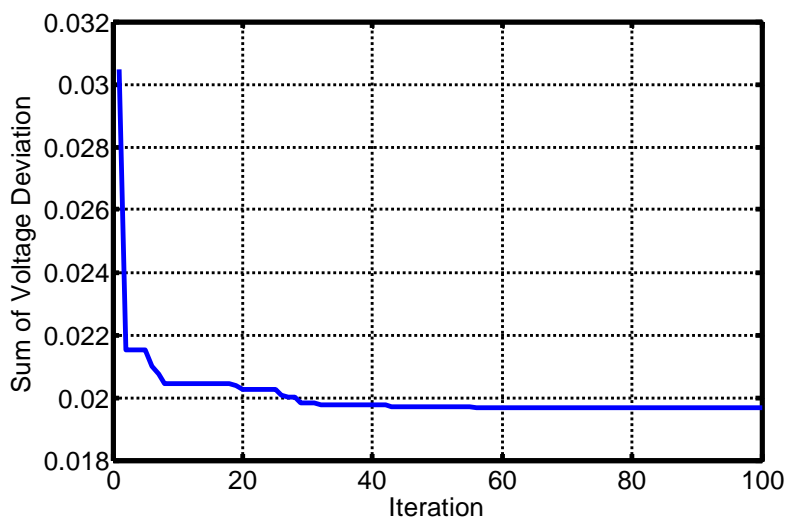

The system voltage profile considering STATCOM device and it is compared to that of case las shown in Fig. 14. It is clear that the voltage profile is greatly improved compared to that of case 1 .

Fig. 13 The voltage deviation variation of case 3

Table 2. Optimal Setting of Control Variables

\begin{tabular}{|c|c|c|c|c|c|}
\hline Variable & Min & Max & Case 1 without FACTS & Case 2 with SVC & Case 3 with STATCOM \\
\hline $\mathrm{P}_{1}$ & 0.50 & 2.00 & 1.7773 & 1.7614 & 1.7165 \\
\hline $\mathrm{P}_{2}$ & 0.20 & 0.80 & 0.4926 & 0.4857 & 0.4758 \\
\hline $\mathrm{P}_{3}$ & 0.15 & 0.50 & 0.2149 & 0.2196 & 0.2103 \\
\hline $\mathrm{P}_{4}$ & 0.10 & 0.35 & 0.2204 & 0.2144 & 0.2001 \\
\hline $\mathrm{P}_{5}$ & 0.10 & 0.30 & 0.1011 & 0.1214 & 0.1000 \\
\hline $\mathrm{P}_{6}$ & 0.12 & 0.40 & 0.1200 & 0.1211 & 0.1200 \\
\hline $\mathrm{V}_{1}$ & 0.95 & 1.10 & 1.0730 & 1.0730 & 1.0837 \\
\hline $\mathrm{V}_{2}$ & 0.95 & 1.10 & 1.0721 & 1.0620 & 1.0685 \\
\hline $\mathrm{V}_{3}$ & 0.95 & 1.10 & 1.0175 & 1.0385 & 1.0453 \\
\hline $\mathrm{V}_{4}$ & 0.95 & 1.10 & 1.0557 & 1.0433 & 1.0527 \\
\hline $\mathrm{V}_{5}$ & 0.95 & 1.10 & 1.0462 & 1.1165 & 1.1429 \\
\hline $\mathrm{V}_{6}$ & 0.95 & 1.10 & 0.9897 & 0.9997 & 1.0586 \\
\hline $\mathrm{T}_{9-13}$ & 0.90 & 1.10 & 0.9785 & 0.9785 & 0.9785 \\
\hline $\mathrm{T}_{10-13}$ & 0.90 & 1.10 & 1.0875 & 1.0875 & 1.0875 \\
\hline $\mathrm{T}_{8-12}$ & 0.90 & 1.10 & 1.0125 & 1.0125 & 1.0125 \\
\hline $\mathrm{T}_{28-27}$ & 0.90 & 1.10 & 1.0125 & 1.0125 & 1.0125 \\
\hline $\mathrm{Q}_{\mathrm{svc}} \quad$ p.u. & 0.00 & 0.50 & -- & 0.2618 & -- \\
\hline Q $_{\text {STAтсом }}$ p.u. & 0.00 & 0.50 & -- & -- & 0.2392 \\
\hline Fuel cost $\$ / h$ & & & 801.1115 & 800.627 & 765.8002 \\
\hline $\mathrm{P}_{\text {loss }} \quad \mathrm{MW}$ & & & 3.2000 & 3.1200 & 2.9200 \\
\hline$\sum$ Voltage deviation & & & 0.0310 & 0.0275 & 0.0197 \\
\hline
\end{tabular}




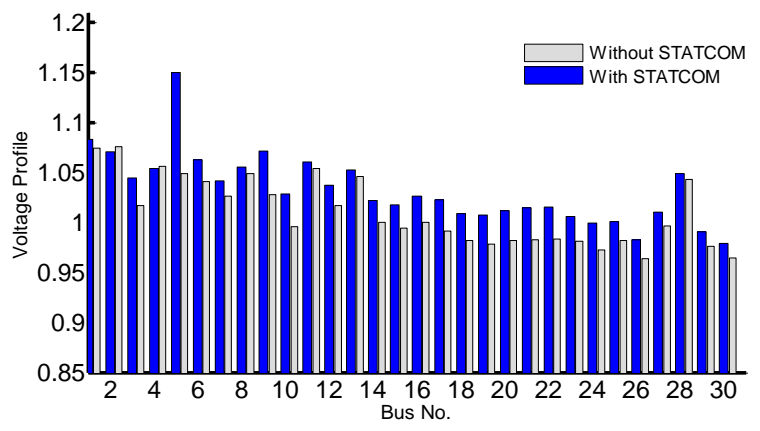

Fig. 14 The system voltage profile considering STATCOM Table 2 illustrates the optimal setting of control variables, also it shows the obtained results for different three cases under study.

For the purpose of verifying the efficiency of the proposed approach, we made a comparison of our approach with others competing OPF algorithm. The computed fuel cost by a non-linear programming method (NLP) 802.40 (\$/h), in an evolutionary programming (EP) it is $\mathbf{8 0 2 . 6 2}(\mathbf{\$} / \mathbf{h})$, in a tabu search (TS) algorithm is $\mathbf{8 0 2 . 2 9}(\mathbf{\$} / \mathbf{h})$, in an improved evolutionary algorithm (IEP) it is $\mathbf{8 0 2 . 4 6 5}(\mathbf{\$} / \mathbf{h})$, and in GA-Fuzzy system approach it is $\mathbf{8 0 2 . 0 0 3} \mathbf{( \$ / h )}$. The operating cost in our approach proposed is $\mathbf{8 0 1 . 1 1 1 5}$ (\$/h) and the power loss is $\mathbf{3 . 2 0}$ (MW) which are better than the others methods reported in the literature [18].

From the recorded results in Table 2, it can be concluded that using old type of FACTS such SVC will improve the system performance where can minimize each of total fuel cost, network power losses and summation of voltage deviation. Also, it is clear that using advanced type of FACTS such STATCOM can minimize each of the total fuel cost, system power losses and summation of voltage deviation much more compared with SVC.

\section{Conclusion}

A proposed Particle Swarm Optimization based OPF problem has been presented. The proposed approach utilizes the global and local exploration capabilities of PSO to search for optimal setting of control variables. The PSO algorithm is preferred compared with each of GA/Fuzzy, EP, TS and IEP methods. Two different types of FACTS devices (SVC and STATCOM) have been employed to minimize each of the total fuel cost, system power losses and improve the system voltage profile. The simulation results illustrate the effectiveness of the proposed STATCOM with optimal settings compared with SVC in terms of reduced total fuel cost, power loss and voltage deviation.

\section{REFERENCES}

[1] N.R. Hasma Abdullah, I. Musirin and M. Othman, "Transmission Loss Minimization and UPFC Installation Cost using Evolutionary Computation for Improvement of Voltage Stability", Proceedings of the 14th International Middle East Power Systems Conference (MEPCON'10), Cairo University, Egypt, Paper ID 293 December 19-21, 2010.

[2] H.R. Baghace, M. Jannati, B. Vahid, S.H. Hosseininan and S. Jazebi, "Optimal multi-type FACTS Allocation using Genetic Algorithm to Improve Power System Securty", IEEE conference, pp. 162-166, 2008.

[3] K. Venkateswarlu and CH. Saibabu, "A New Evolutionary Algorithms Used for Optimal Location of UPFC on Power System", Journal of Theoretical and Applied Information Technology, JATIT \& LLS. All rights reserved, pp. 107-118, ( 2010.

[4] S. Kamel, M. Abdel-Akher, and M. K. El-Nemr, "Implementation of SSSC Model in the NewtonRaphson Power Flow Formulation Using Current Injections", UPEC $31^{\text {st }}$ Aug. - $3^{\text {rd }}$ Sept 2010.

[5] F. B. Alhasawi, J. V. Milanović, A. A. Alabduljabbar, "Economic Viability of Application FACTS Devices for Reducing Generating Costs", IEEE conference pp. 2-8, 2010.

[6] P. Smita, B.N .Vaidya, "Optimal Power Flow by Particle Swarm Optimization for Reactive Loss Minimization", International Journal of Scientific \& Technology Research Vol. 1, Issue 1, pp. 1-6, (IJSTR@2012) Feb. 2012.

[7] B. Mahdad, T. Bouktir and K. Srairi, "Optimal power Flow of the Algerian Network using Genetic Algorithm/Fuzzy Rules”, IEEE con. 2008.

[8] J. G. Vlachogiannis and K. Y. Lee, Fellow, IEEE, "A Comparative Study on Particle Swarm Optimization for Optimal Steady-State Performance of Power Systems", IEEE Transaction power systems, Vol. 21, no. 4, pp. 1718-1728, November 2006.

[9] M. Peikherfeh, M. Abapour, M. Parsa Moghaddam, Member, IEEE, A. Namdari, "Optimal Allocation of FACTS Devices for Provision of Voltage Control Ancillary Services", IEEE con. 2010.

[10] M.A. Abido, "Optimal Power Flow Using Particle Swarm Optimization", Electrical Power and Energy System 24, pp. 562-571, 2002.

[11] G.K. Venayagamoorthy, et al, "Effect of a STATCOM, a SCRC and a UPFC on the Dynamic Behavior of a 45 Bus Seaction of Barazilian Pwer System", Inaugural IEEEPES 2005 Conference and Exposition in Africa Durban, South Africa, pp. 305-312, 11-15 July, 2005.

[12] R. Palma-Behnke, Luis S. Vargas, J. R. Pérez, J. D. Núñez, and Rigoberto A., "OPF With SVC and UPFC Modeling for Longitudinal Systems", Torres IEEE Transactions on Power Systems, Vol. 19, No. 4, November 2004. 
[13] R. Sirjani, A. Mohamed and H. Shareef, “Optimal Placement and Sizing of Shunt FACTS Devices in Power Systems Using Heuristic Optimization Techniques", ISSN 0033-2097, R. 88 NR, pp. 335341, 2012.

[14] V. Singh, Ch. Jitendra, Meel T Jayabarathi, "Optimal Location of STATCOM on Transmission Network using Evolutionary Algorithms", International Journal of Computer Applications, Volume 45-No.19, pp. 36-41, May 2012.

[15] N. Mancer, B. Mahdad and K. Srairi, "Multi Objective Optimal Reactive Power Flow Based STATCOM Using Three Variant of PSO", International Journal of Energy Engineering 2012.

[16] J. Namratha Hamanohar and J. Amarnath, "Performance Enhancement of Power System by STATCOM-Integrated Architecture", International Journal of Electrical and Electronics Engineering (IJEEE), ISSN (PRINT): 2231 - 5284 Vol. 1 Issue 4, pp. 41-46, 2012.

[17] M. Saad S. Al-ghamdi, "Multi-Objective Optimal Power Flow", Master of Science in Electrical Engineering, May 2004.

[18] B. Mahdad, K. Srairi, T. Bouktir and M. EL. Benbouzid, "Optimal Power Flow with Discontinous Fuel Cost Functions Using Decomposed GA Coordinated with Shunt FACTS”, Journal of Electrical Engineering \& Technology Vol. 4, pp. 457-466, No. 4, 2009. 
Ekramy Saad, R. A. Amer and G. A. Morsy " Optimal Power Flow Control Based Shunt FACTS Devices ......." 